ARTICLE

https://doi.org/10.1038/s41467-019-09745-2

\title{
Clonal evolution patterns in acute myeloid leukemia with NPM1 mutation
}

Sibylle Cocciardi ${ }^{1}$, Anna Dolnik , Silke Kapp-Schwoerer ${ }^{1}$, Frank G. Rücker ${ }^{1}$, Susanne Lux', Tamara J. Blätte ${ }^{1}$, Sabrina Skambraks', Jan Krönke, Florian H. Heidel ${ }^{2,3}$, Tina M. Schnöder ${ }^{2,3}$, Andrea Corbacioglu', Verena I. Gaidzik 1 , Peter Paschka', Veronica Teleanu', Gudrun Göhring ${ }^{4}$, Felicitas Thol ${ }^{5}$, Michael Heuser ${ }^{5}$, Arnold Ganser ${ }^{5}$, Daniela Weber ${ }^{1}$, Eric Sträng ${ }^{6}$, Hans A. Kestler ${ }^{1}{ }^{6}$, Hartmut Döhner ${ }^{1}$, Lars Bullinger ${ }^{1,7,8}$ \& Konstanze Döhner ${ }^{1,8}$

Mutations in the nucleophosmin 1 (NPM1) gene are considered founder mutations in the pathogenesis of acute myeloid leukemia (AML). To characterize the genetic composition of NPM1 mutated (NPM7mut) AML, we assess mutation status of five recurrently mutated oncogenes in 129 paired NPM7mut samples obtained at diagnosis and relapse. We find a substantial shift in the genetic pattern from diagnosis to relapse including NPM7mut loss ( $n=$ 11). To better understand these NPM7mut loss cases, we perform whole exome sequencing (WES) and RNA-Seq. At the time of relapse, NPM7mut loss patients (pts) feature distinct mutational patterns that share almost no somatic mutation with the corresponding diagnosis sample and impact different signaling pathways. In contrast, profiles of pts with persistent NPM $7^{m u t}$ are reflected by a high overlap of mutations between diagnosis and relapse. Our findings confirm that relapse often originates from persistent leukemic clones, though NPMTmut loss cases suggest a second "de novo" or treatment-associated AML (tAML) as alternative cause of relapse.

\footnotetext{
${ }^{1}$ Department of Internal Medicine III, University Hospital of UIm, UIm 89081, Germany. ${ }^{2}$ Department of Internal Medicine II, Hematology and Oncology, Friedrich-Schiller-University Medical Center, Jena 07743, Germany. ${ }^{3}$ Leibniz-Institute on Aging, Fritz-Lipmann-Institute, Jena 07745, Germany. ${ }^{4}$ Institute of Cell \& Molecular Pathology, Hannover Medical School, Hannover 30625, Germany. ${ }^{5}$ Department of Haematology, Haemostasis, Oncology, and Stem Cell Transplantation, Hannover Medical School, Hannover 30625, Germany. ${ }^{6}$ Institute of Medical Systems Biology, Ulm University, Ulm 30625, Germany. ${ }^{7}$ Department of Hematology, Oncology and Tumorimmunology, Charité University Medicine, Berlin 13353, Germany. ${ }^{8}$ These authors contributed equally: Lars Bullinger, Konstanze Döhner. Correspondence and requests for materials should be addressed to L.B. (email: lars.bullinger@charite.de) or to K.D. (email: konstanze.doehner@uniklinik-ulm.de)
} 
O ne of the most common mutations (mut) in AML involves the NPM1 gene, which is present in about one third of AML pts ${ }^{1}$. NPM1 $1^{\text {mut }}$ AML is characterized by distinct biological and clinical features and pts with the NPM1 ${ }^{\text {mut }}$ and no FLT3 internal tandem duplication (ITD) or low FLT3ITD levels have a good response to induction chemotherapy and a favorable prognosis. Based on these findings $N P M 1^{\text {mut }} \mathrm{AML}$ was included as an entity in the World Health Organization classification 2016 and the NPM1 ${ }^{\text {mut }} / F L T 3-I T D$ genotypes stratified by the ITD allelic ratio were integrated in the risk stratification of the European LeukemiaNet (ELN) recommendations, subdividing AML in subsets with highly distinct prognosis ${ }^{2-4}$.

For a long time $N P M 1^{\text {mut }}$ was considered a founder event, because it is usually maintained at relapse. The consideration of $N P M 1^{\text {mut }}$ as a founder event in AML is further supported by the distinct morphological and clinical presentation associated with this subtype of AML. However, recent studies have shown that $N P M 1^{\text {mut }}$ occurs rather late, due to its absence in preleukemic hematopoietic stem cells (HSCs) ${ }^{5,6}$. Moreover, in $\sim 10 \%$ of relapsed pts $N P M 1^{\text {mut }}$ is lost while further chromosomal and molecular changes are acquired ${ }^{7-9}$.

The recently identified preleukemic mutations in DNMT3A, TET2, ASXL1, IDH1, and IDH2 often persist at remission due to persistent clonal hematopoiesis ${ }^{5,10}$. Preleukemic mutations were also found to be present in non-leukemic T cells of AML patients, at the time of diagnosis and they have been identified in individuals without hematologic malignancy or who were unselected for cancer or hematologic phenotypes in an age related-manner ${ }^{11}$. Age-related clonal hematopoiesis is a common condition that is associated with an increased risk to develop hematologic cancer $^{12-15}$. Indeed, cooccurring mutations in DNA methylation or hydroxymethylation genes (DNMT3A,IDH1,IDH2R140, and TET2) are frequent amongst $N P M 1^{\text {mut }}$ leukemias and found in $\sim 73 \%$ of $\mathrm{pts}^{6}$.

In our previously investigated cohort of $53 N P M 1^{\text {mut }}$ AML pts, we also described this frequent co-occurrence of preleukemic mutations ${ }^{7}$. Here, the majority of relapsed leukemias showed clonal evolution with a clear relationship of relapse and diagnostic leukemia clones by acquisition of additional genetic lesions, or more commonly the relapse clone arose from a common ancestral clone, which was also shown by other studies ${ }^{7,16,17}$. Interestingly, in our study we identified five pts with NPM1 ${ }^{\text {mut }}$ loss at relapse showing a shift in genetic lesions between the diagnosis and relapse sample; clinically, these patients had a significantly longer time to relapse compared to $N P M 1^{\text {mut }}$ persistent pts (33.7 months versus 8.6 months, $p=0.03$ ), and none responded to salvage therapy. To date, it is still unclear whether in these cases clonal evolution resulted in loss of $N P M 1^{\text {mut }}$ or whether the initial leukemia clone was cured and a new leukemia developed on the basis of dominant clonal hematopoiesis with persisting preleukemic mutations. This new leukemia could then also be considered as therapy-related AML (tAML) that evolved from a common pre-leukemic clone.

To gain further insight into the genomic evolution of $N P M 1^{\text {mut }}$ AML, we have extended our previous cohort and assess mutational status of five genes by conventional mutation analysis in paired samples obtained at diagnosis and relapse from $129 \mathrm{NPM1}{ }^{\text {mut }}$ pts. For a subgroup of pts a more detailed analysis comprising the mutational status of nine genes and comprehensive SNP microarray profiling is performed. Finally, for in-depth analysis we sequence the exome of ten pts with persistent NPM1 $1^{\text {mut }}$ and ten pts with loss of $N P M 1^{\text {mut }}$ and perform RNA-Seq analysis for selected $N P M 1^{\text {mut }}$ persistent and loss cases. Findings are confirmed by targeted deepsequencing and flow cytometry based single cell protein expression analysis.

\section{Results}

Genomic characterization of clonal evolution in $N P M 1^{\text {mut }}$ AML. Paired samples at diagnosis and relapse from 129 NPM1 $1^{\text {mut }}$ AML pts were assessed for clonal evolution-associated mutations in the most recurrently mutated genes (FLT3, DNMT3A, IDH1, IDH2, NRAS) by conventional mutation analysis as previously reported ${ }^{7}$. At diagnosis, 83 pts (64\%) harbored concurrent DNMT3A ${ }^{\text {mut }}$, 40 pts (31\%) FLT3-ITD, 22 pts (17\%) FLT3-tyrosine kinase domain (TKD) ${ }^{\text {mut }}, 23$ pts (18\%) NRAS ${ }^{\text {mut }}, 29$ pts (23\%) and 24 pts $(19 \%) I D H 1^{\text {mut }}$ and $I D H 2^{\text {mut }}$, respectively (Fig. 1). In addition, we screened a subgroup of pts for $M L L$ partial tandem duplications (PTD) and mutations in ASXL1, TP53 and RUNX1. None of the pts analyzed had a $M L L-P T D$, ASXL1 $1^{\text {mut }}, T P 53^{\text {mut }}$ or $R U N X 1^{\text {mut }}$ at the time of diagnosis (Supplementary Fig. 1 and Supplementary Table 1).

At relapse, a shift in the mutation pattern was found in 76 pts (59\%, Supplementary Table 2). While $N P M 1^{\text {mut }}$ was lost in 11 pts (9\%), DNMT3A $A^{\text {mut }}$ persisted in 79 of 83 pts $(95 \%)$ and thus was the most stable mutation at relapse. Sixteen of 40 pts $(40 \%)$ had the identical FLT3-ITD, whereas 14 pts (35\%) showed a distinct FLT3ITD clone indicated by a change of the ITD length, and 10 pts (25\%) lost the FLT3-ITD at relapse. Gain of a FLT3-ITD clone was detected in 23 of 128 cases (18\%). Similarly, FLT3-TKD ${ }^{\text {mut }}$ and NRAS ${ }^{\text {mut }}$ were rather unstable and lost in 16 of 22 pts (73\%) and 15 of 23 pts (65\%), respectively. On the contrary, $I D H 1^{\text {mut }}$ and

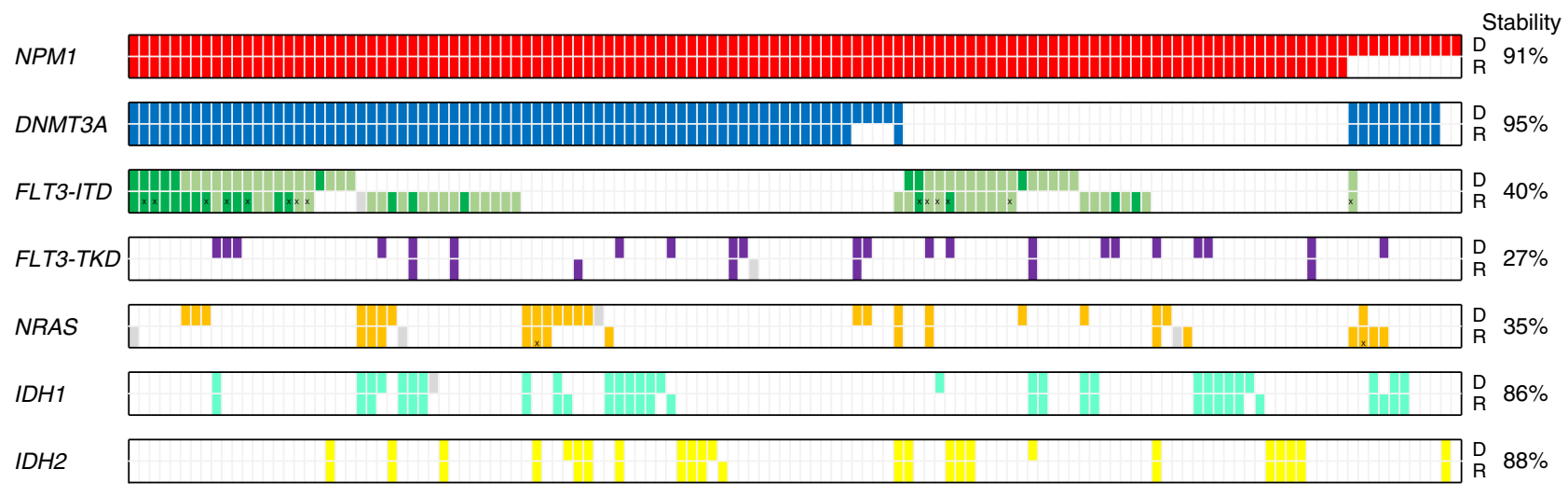

Fig. 1 Incidence of mutations in 129 paired (diagnosis/relapse) NPM7mut pts. Colored bars indicate the presence of a mutation, white bars represent wildtype, data not available is indicated by a gray bar. Light and dark green bars illustrate heterozygous and homozygous FLT3-ITD mutations, respectively. Bars marked by $X$ illustrate different mutation types found in the diagnosis and relapse sample. Stability was calculated by the number of mutations that persisted at relapse divided by all mutations present at diagnosis. $D$, diagnosis; $R$, relapse 


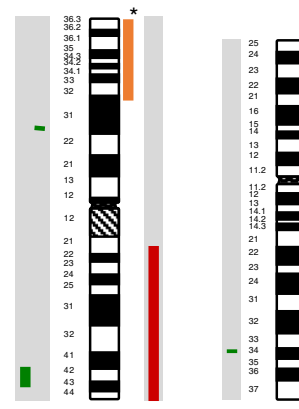

1

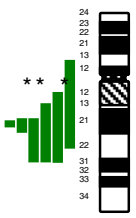

9

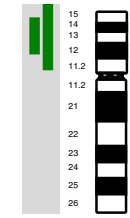

10
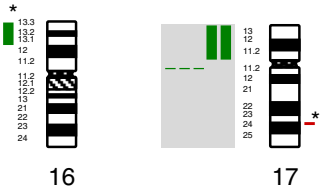

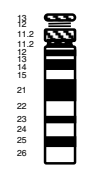

15

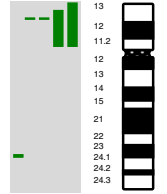

12

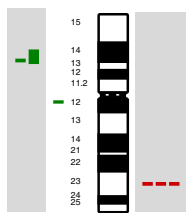

11

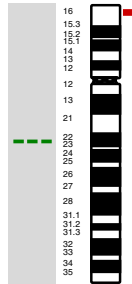

4

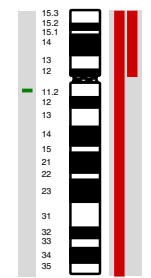

5

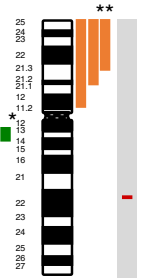

- Loss

- Gain

- UPD

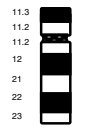

18

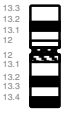

19

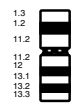

20

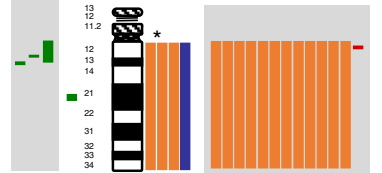

13

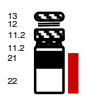

21

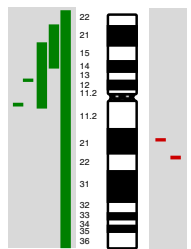

7

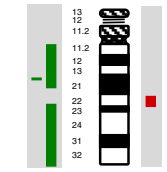

14

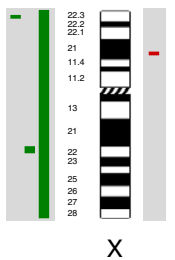

Fig. 2 CNAs and UPDs identified at diagnosis and relapse by SNP profiling. Bars next to each chromosome indicate genomic gain, loss, and uniparental disomy (UPD). The length of the bar indicates the respective genomic region affected by the aberrations. Bars highlighted by a grey box indicate relapse specific aberrations. Bars marked by a star indicate genomic changes which are lost at relapse

$\mathrm{IDH} 2^{\text {mut }}$ were relatively stable, with persistence of the diagnosis mutation in 25 of 29 pts (86\%) for $I D H 1^{\text {mut }}$ and 21 of 24 pts $(88 \%)$ for $I D H 2^{\text {mut }}$. For $M L L-P T D, A S X L 1^{\text {mut }}$, TP53 $3^{\text {mut }}$ and RUNX1 ${ }^{\text {mut }}$, none of the pts with $N P M 1^{\text {mut }}$ loss had a mutation at diagnosis; however, at relapse four pts gained an $M L L-P T D$, one an $A S X L 1^{\text {mut }}$, and three a $R U N X 1^{\text {mut }}$ (Fig. 1 and Supplementary Fig. 1).

Following up on our previous report ${ }^{7}$, we extended single nucleotide polymorphism (SNP) microarray profiling to a total of $77 N P M 1^{\text {mut }}$ pts [cytogenetically normal $(\mathrm{CN})$ karyotype, $n=73$; $\operatorname{del}(9 \mathrm{q}), n=4]$. In addition to these findings also seen by cytogenetics, the analysis of diagnostic samples confirmed copy number alterations (CNAs) and uniparental disomy (UPD) previously associated with $\mathrm{CN} N P M 1^{\text {mut }}$ including $\operatorname{del}(4)$ (q22.1), del(12)(p13.2), del(17)(q11.2), gain of chromosome 8 (trisomy 8) and UPD affecting chromosomal arm 13q (Fig. 2 and Supplementary Table 3). In the extended cohort, novel findings at diagnosis included del(11)(q12.3), gain(8)(q24.21), and a trisomy 21 as well as UPDs affecting the chromosomal arm $6 \mathrm{p}$ in 3 pts (Fig. 2). At relapse, the previously reported deletions of $12 \mathrm{p} 13$ and 17q11.2, gain(11)(q23.3), trisomy 8 and UDP 13 could also be confirmed. In addition, SNP-array analysis identified nine non-recurrent CNAs [del(5)(q11.2q11.2), $\operatorname{del}(10)(\mathrm{p} 11.21 \mathrm{p} 15.3)$, $\operatorname{del}(10)(\mathrm{p} 12.31 \mathrm{p} 14), \quad \operatorname{del}(14)(\mathrm{q} 11.2 \mathrm{q} 21.3), \quad \operatorname{del}(14)(\mathrm{q} 23.1 \mathrm{q} 32.33)$, $\operatorname{del}(\mathrm{X})(\mathrm{p} 22.32 \mathrm{q} 26.1), \quad$ gain(1)(q21.1q44), gain(5)(p11p15.33), gain(14)(q22.1q22.2)], as well as one UPD affecting the long arm of chromosome 21 (Fig. 2). The incidence of chromosomal aberrations (deletion, gain, or UPD) was 3.4 times higher at relapse (diagnosis $n=23$, relapse $n=79$ ) as shown previously ${ }^{7}$.

Overall, we detected a gain of CNAs and/or UPDs at relapse in $66.7 \%(4 / 6)$ of $N P M 1^{\text {mut }}$ loss and $43.7 \%(31 / 71)$ of $N P M 1^{\text {mut }}$ persistent samples. Patients with gain of CNA and/or UPD also acquired more mutations at relapse based on mutations detected with WES (median 7.5 vs 2; pts with gain of CNA and/or UPD, $n=8$; pts without gain of CNA and/or UPD, $n=5)$. This supports our observation that $N P M 1^{\text {mut }}$ loss pts are less stable in terms of their mutational profile compared to $N P M 1^{\text {mut }}$ persistent pts. Interestingly, 10/11 NPM1 $1^{\text {mut }}$ loss pts were in the favorable ELN 2017 risk group at the time of diagnosis while only one was in the intermediate risk group.

Comparative analysis of NPM1 ${ }^{\text {mut }}$ loss and persistent pts. To gain further insight into the relapse mechanism of pts with loss of the $N P M 1^{\text {mut }}$, we performed WES of paired samples (diagnosis, remission and relapse) from $10 \mathrm{NPM} 1^{\text {mut }}$ loss and $10 \mathrm{NPM} 1^{\text {mut }}$ persistent pts. The mean depth of the covered exome was 91 reads for diagnosis, 92 reads for remission, and 89 reads for relapse samples (Supplementary Table 4). At diagnosis, we identified on average 9.7 mutations per case (8.8 in NPM1 $1^{\text {mut }}$ loss and 10.6 in $N P M 1^{\text {mut }}$ persistent pts) and at relapse 11.4 mutations per case (9.4 in $N P M 1^{\text {mut }}$ loss and 13.4 in $N P M 1^{\text {mut }}$ persistent pts).

In all $N P M 1^{\text {mut }}$ loss pts, WES identified at least one previously described preleukemic mutation with the majority carrying DNMT3A $A^{\text {mut }}(9 / 10 \text { pts, Fig. 3a and Supplementary Data } 1)^{5,11,12}$. Notably, all preleukemic mutations persisted with a similar variant allele frequency (VAF) in remission and relapse (Supplementary Fig. 2a). In addition, we identified mutations persisting at remission in genes not yet associated with clonal hematopoiesis such as $P T K 2 B$ and PAX5. Apart from these persisting mutations, diagnosis and relapse samples had distinct mutational patterns and shared almost no somatic mutation. Thus, in NPM1 ${ }^{\text {mut }}$ loss pts not only $N P M 1^{\text {mut }}$ but also all other somatic mutations were lost, thereby 
a

\begin{tabular}{|c|c|c|c|}
\hline \multicolumn{4}{|c|}{$N P M 1^{\text {mut }}$ loss } \\
\hline ID & Diagnosis & Remission & Relapse \\
\hline 12 & $\begin{array}{l}\text { DNMT3A, IDH1 } \\
\text { NPM1, CPNEB, CSAD, NRG2, OGT, PTPN11, ZKSCAN2, ZNF211 }\end{array}$ & DNMT3A, IDH1 & $\begin{array}{l}\text { DNMT3A, IDH1 } \\
\text { BSN, FBN1, MED 12, MNDA, NRAS, PRPF8, SLCGA5 }\end{array}$ \\
\hline 103 & $\begin{array}{l}\text { DNMTA, PTK2B } \\
\text { NPM1. AMT, FLTS-ITD* HTR3A, MCF2L, MYPN }\end{array}$ & DNMT3A, PTK2B & $\begin{array}{l}\text { DNMT3A, PTK2B } \\
\text { AMDHD1, AP2A1, DCX, FLTT-ITD* GIGYF1, ICA1, NRAS, WDR53, WT1 }\end{array}$ \\
\hline 123 & $\begin{array}{l}\text { DNMT3A, RUNX1, TET2, TET2, TET2 } \\
\text { NPM1, ANKRD 13D, BCAN, CARS2, CST11, NMI }\end{array}$ & $\begin{array}{l}\text { DNMT3A, RUNX1, } \\
\text { TET2, TET2, TET2 }\end{array}$ & $\begin{array}{l}\text { DNMT3A, RUNX1, TET2, TET2, TET2 } \\
\text { NMI, THSDA, ZFYVE1 }\end{array}$ \\
\hline 172 & $\begin{array}{l}\text { DNMT3A } \\
\text { NPM1, ANO1, FIGN, ITGA11, KIF14, LRRN3, NRAS", PTPN11* }\end{array}$ & DNMT3A & $\begin{array}{l}\text { DNMT3A } \\
\text { ATM, KRTAP-32, NRAS: PTPN11; RUNX1 }\end{array}$ \\
\hline 355 & $\begin{array}{l}\text { DNMT3A, IDH1 } \\
\text { NPM1, BHMT2, FSD1, HIPK1, KAT6B, PDLIM1, PHF12, PSTPIP2, TOP2B, ZBTB47 }\end{array}$ & DNMT3A, IDH1 & $\begin{array}{l}\text { DNMT3A, DNMT3A, IDHT, TET1 } \\
\text { ARNT, ASXL3, , RAA, CACNA1E, CHUK, CNTRL, CYLC1, DMBT1, FAT2, FBXO28, FZDE, IFNE, LINGO4, } \\
\text { MAP2K3, MDN1, NF1, NPAT, PARS2, PTPRO, ZCCHC6 }\end{array}$ \\
\hline 524 & $\begin{array}{l}\text { DNMT3A } \\
\text { NPMM1, ADAM18, FLT3-TKD, LZTR1, MAPK10, PILRA, SEC24C, SMC1A, XDH }\end{array}$ & DNMT3A, IDH1 & $\begin{array}{l}\text { DNMT3A, IDH1 } \\
\text { CAMKK1, HIST1HBB, MANSC4, NRAS, PARDBB, PTCHD2, SLCGA1, TRAK1, WIPF2 }\end{array}$ \\
\hline 1214 & $\begin{array}{l}\text { DNMT3A, PAX5 } \\
\text { NPM1, ANTXR1, CATSPERB, FOXA3, SYCP2, USH2A }\end{array}$ & DNMT3A, PAX5 & $\begin{array}{l}\text { DNMT3A, PAX5 } \\
\text { ARMC8, TMC7, CACNA1C, ABCB11, CWC22, DLG1, ZHX2, RAB17, WT1, WT1 }\end{array}$ \\
\hline 6013 & $\begin{array}{l}\text { DNMT3A } \\
\text { NPM1, KHDRBS2, LTBP1, NOTCH3, RBL1 }\end{array}$ & DNMT3A & $\begin{array}{l}\text { DNMT3A } \\
\text { TRPV4 }\end{array}$ \\
\hline 6018 & $\begin{array}{l}\text { BCOR, IDH2 } \\
\text { NPM1, CBFA2T3, DNAJC24 }\end{array}$ & $B C O R, I D H 2$ & $\begin{array}{l}\text { BCOR, IDH2 } \\
\text { CBFA2T3, DNASE2, ESF1, FBXO9, PHF3, PXDNL, RUNX1 }\end{array}$ \\
\hline 6034 & $\begin{array}{l}\text { DNMT3A } \\
\text { NPM1, ATG16L2, ATP13A1, CABP1, CASP6, CTAGE5, CYP4F8, DEF6, DNAJB12, EIF2AK4, INHBE, MAPF, MPP2, } \\
\text { NARF, RGL3, SDK1, SFXN4, TMEM170B, ZCWPW1 }\end{array}$ & DNMT3A & $\begin{array}{l}\text { DNMT3A } \\
\text { CYP4F8, ZFHX3, ZNF267 }\end{array}$ \\
\hline \multicolumn{4}{|c|}{$N P M 1^{\text {mut }}$ persistent } \\
\hline ID & Diagnosis & Remission & Relapse \\
\hline 31 & $\begin{array}{l}\text { DNMT3A } \\
\text { NPM1, ETS1, GPHA2, NCDN, MYBPHL, PTPN11, RAD21, TTC28, TUBGCP6 }\end{array}$ & & NPM1, GPHA2, MAT2A, TTC28, TUBGCP6 \\
\hline 194 & $\begin{array}{l}\text { DNTI3A } \\
\text { NPM1. ANKK1, FLT3-ITD, HERC2, SDR16C5, SH2B3, TMC1 }\end{array}$ & DNMT3A & $\begin{array}{l}\text { ASX11, DNMT3A } \\
\text { NPM1, FLT3-ITD, LMX1B, MBOAT1, SH2B3, SPTBN2, TMEM18, VPS11, ZC3H14 }\end{array}$ \\
\hline 340 & $\begin{array}{l}\text { DNMT3A } \\
\text { NPM1. ARSG, ERBB2, FBLIM1, FLT3-ITD, LAMB4, NCOR1, NLGN1, PLEKHS1, SULF2, UNC5D, ZNF160, WT1 }\end{array}$ & DNMT3A & $\begin{array}{l}\text { DNMT1A } \\
\text { NPM1, ARSG, CLSTN1, FBLIM1, FLT3-ITD, LAMB4, NCOR1, NLGN1, PLEKHS1, SULF2, TP53, TRIM24, } \\
\text { UNCSD, UTRN, ZNF160, WT1 }\end{array}$ \\
\hline 509 & NPAT & NPAT & 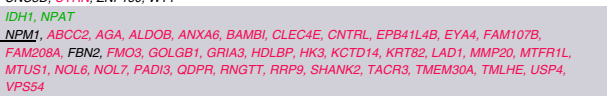 \\
\hline 584 & $\begin{array}{l}\text { DNMT3A } \\
\text { NPM1, FLT3, KAT6A, KRAS, MEIS2, MORC4, PABPC4, PCDHA3, PTPN11, PTPRF, STXBP5L, THBD, ZBTB18 }\end{array}$ & DNMT3A & $\begin{array}{l}\text { DNMT3A, IDH1 } \\
\text { NPM1, ANGEL1, CDK16, CNTNAP2, KATGA,KIT, MEIS2, NMU, PCDHA3, PTPRF, PTPRZ1, SMEK2, } \\
\text { STXBPSL, TTN, ZBTB18 }\end{array}$ \\
\hline 659 & $\begin{array}{l}\text { DNMT3A, TET2 } \\
\text { NPM1, HIST1H2AA, MYO18B, SPEN, TENM4, ZNF473 }\end{array}$ & DNMT3A, TET2 & $\begin{array}{l}\text { DNMT3A, TET2 } \\
\text { NPM1, CACNA1E, HDHD3, HIST1H2AA, MYO18B, PLA2G3, SPEN, TENM4, ZNF473 }\end{array}$ \\
\hline 752 & $\begin{array}{l}\text { DNMT3A, IIH1 } \\
\text { NPM1, BRINP1, CCDC168, CDK18, COL3A1, DCLK3, FAM179A, FLT3, LPHN3, RBM41 }\end{array}$ & DNMT3A & $\begin{array}{l}\text { DNMT3A, IDH1 } \\
\text { NPM1, BRINP1, CCDC168, CDK18, COL3A1, DCLK3, FAM179A, FLT3,LPHN3, MYD88, PDE8A, RBM41 }\end{array}$ \\
\hline 856 & $\begin{array}{l}\text { DNMM 3A, IDH2 } \\
\text { NAAV1, ADAMTS3, COL12A1, CYPAB1, FKBPG, KRAS, LRAC16B, NRAS, ORS6A4, OR7G2, PCDHB14, TLE2, }\end{array}$ & & $\begin{array}{l}\text { DNMT1, IDAM } \\
\text { NPAV1 ADAMTS3, COL12A1, CYPAB1, DAAM2, NRAS, OR56A4, OR7G2, PCDHB14, RRC16B, SPAG4, }\end{array}$ \\
\hline 858 & $\begin{array}{l}\text { DNMT3A } \\
\text { NPM1, GCNT3, KRAS, MYO6, NANOS3, NSD1, PDAP1, PLEKHS1, PP2D1, RAD21, ZRSR2 }\end{array}$ & & NPM1, GCNT3, MYO6, NSD1, PLEKHS1, PP2D1, RAD21 \\
\hline 1019 & $\begin{array}{l}\text { DNMT3A, IDH2 } \\
\text { NPM1, CCDC28B, FAT3, MOB1B, NRAS, PKDZL1, RBBPG, SIMC1, TNS1, ZFHX4 }\end{array}$ & & 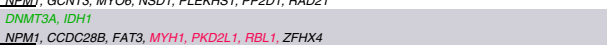 \\
\hline
\end{tabular}

b
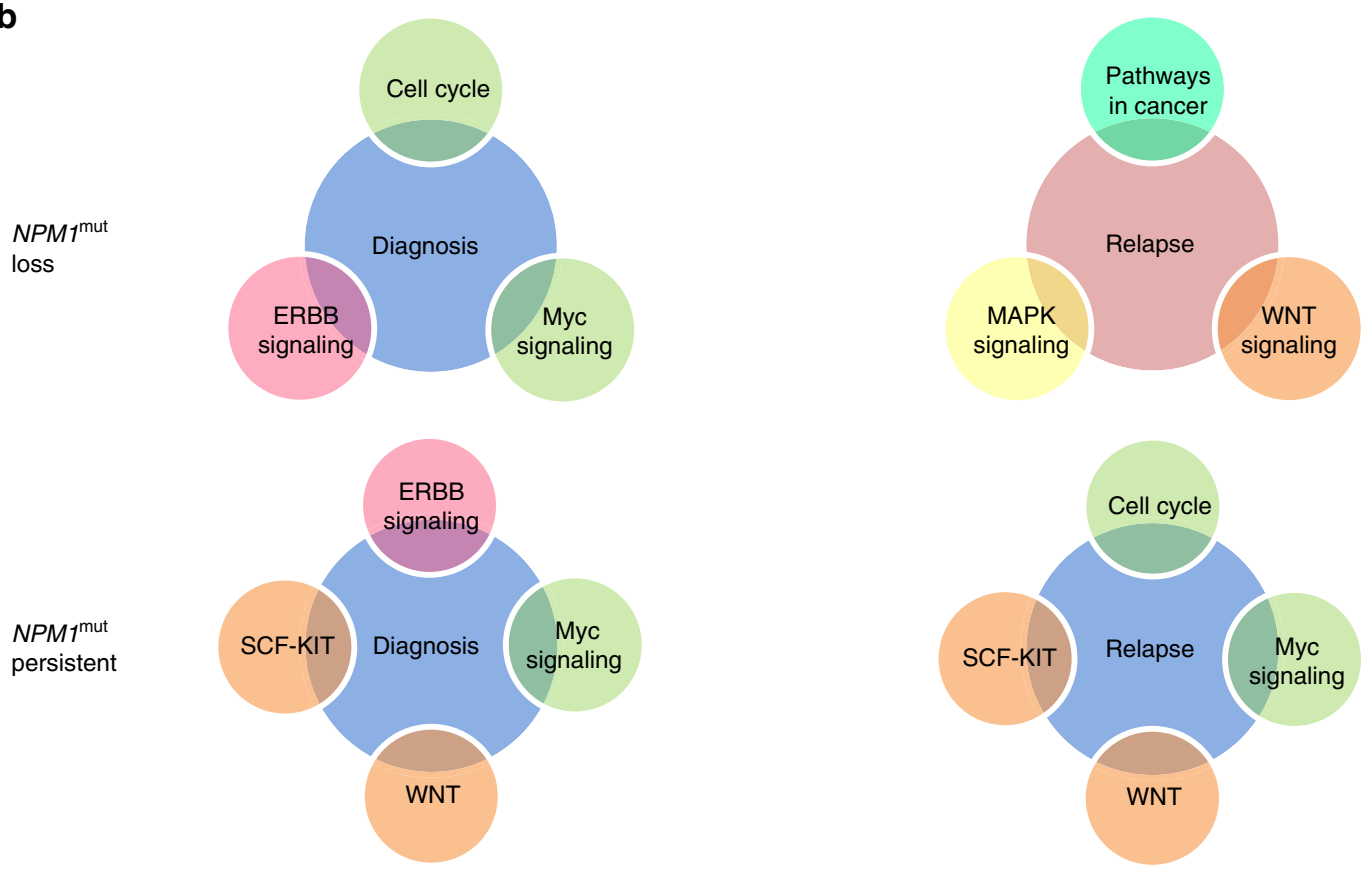

Fig. 3 Mutational map and enriched pathways in diagnosis/relapse pairs of NPM7mut loss and persistent pts. a Mutational profile of 10 NPM7mut loss and 10 NPM7mut persistent pts found by WES. Colors indicate the varying occurrence of mutations during disease progression, including mutations which persist during remission (preleukemic/germline mutations; [green]), mutations which are solely present in the diagnosis sample (blue), relapse specific mutations (red) and mutations which are shared between the diagnosis and relapse sample (black). b Enriched pathways in diagnosis and relapse samples from NPM $7^{\text {mut }}$ loss and NPM ${ }^{\text {mut }}$ persistent pts based on mutated genes 
Table 1 Relapse specific mutations detected in diagnosis samples with UltraDeep-sequencing

\begin{tabular}{|c|c|c|c|c|c|c|c|c|c|c|c|c|c|}
\hline Patient ID & Chr & Start & End & Ref & Alt & Gene & AAChange & $\begin{array}{l}\text { VAF \% } \\
\text { relapse (WES) }\end{array}$ & $\begin{array}{l}\text { VAF \% } \\
\text { remission (WES) }\end{array}$ & $\begin{array}{l}\text { VAF \% } \\
\text { diagnosis (WES) }\end{array}$ & $\begin{array}{l}\text { Found at } \\
\text { diagnosis } \\
\text { (UltraDeep-seq) }\end{array}$ & $\begin{array}{l}\text { Coverage } \\
\text { (UltraDeep- } \\
\text { seq) }\end{array}$ & $\begin{array}{l}\text { VAF \% } \\
\text { (UltraDeep- } \\
\text { seq) }\end{array}$ \\
\hline $103 \mathrm{~L}$ & chr11 & 32417907 & 32417907 & - & CCGA & WT1 & A382fs & 13.54 & 0 & 0 & NO & 9660 & 0 \\
\hline $103 \mathrm{~L}$ & chr1 & 115258744 & 115258744 & $C$ & $\mathrm{~T}$ & NRAS & G13D & 31.89 & 0.45 & 0 & NO & 8062 & 0 \\
\hline $103 \mathrm{~L}$ & chr2 & 25463286 & 25463286 & C & $\mathrm{T}$ & DNMT3A & $\mathrm{R} 736 \mathrm{H}$ & 25 & 37 & 51.35 & YES & 1128 & 29.8 \\
\hline $172 \mathrm{~L}$ & chr12 & 112888211 & 112888211 & A & C & PTPN11 & E76A & 14.29 & 0 & 0 & NO & 5244 & 0 \\
\hline $172 \mathrm{~L}$ & chr21 & 36171600 & 36171600 & - & A & RUNX1 & S322fs & 50 & 0 & 0 & NO & 2207 & 0 \\
\hline $172 \mathrm{~L}$ & chr2 & 25457243 & 25457243 & G & A & DNMT3A & R882C & 38.46 & 36 & 38.46 & YES & 4531 & 48.5 \\
\hline $172 \mathrm{~L}$ & chr1 & 115258744 & 115258744 & C & $\mathrm{T}$ & NRAS & G13D & 8 & 0 & 0 & NO & 9311 & 0 \\
\hline $355 \mathrm{~L}$ & chr7 & 140476755 & 140476755 & $\mathrm{~T}$ & C & BRAF & $1551 \mathrm{~V}$ & 26.47 & 0 & 0 & NO & 2282 & 0 \\
\hline $355 \mathrm{~L}$ & chr2 & 25458596 & 25458596 & $\mathrm{~T}$ & A & DNMT3A & L859F & 35.29 & 0 & 0 & NO & 1163 & 0 \\
\hline $355 \mathrm{~L}$ & chr17 & 29662024 & 29662024 & - & A & NF1 & A1994fs & 36.54 & 0 & 0 & NO & 3309 & 0 \\
\hline $355 \mathrm{~L}$ & $\mathrm{chr} 2$ & 209113113 & 209113113 & G & $\mathrm{T}$ & IDH1 & R132S & 40 & 33 & 43 & YES & 2736 & 45.1 \\
\hline $355 \mathrm{~L}$ & chr2 & 25468187 & 25468187 & A & C & DNMT3A & C497G & 29.27 & 25 & 48.08 & YES & 646 & 44.8 \\
\hline $340 P$ & chr2 & 25457242 & 25457242 & C & $\bar{T}$ & DNMT3A & $\mathrm{R} 882 \mathrm{H}$ & 11 & 7 & 50 & YES & 1172 & 57.3 \\
\hline $340 P$ & chr5 & 170837543 & 170837543 & - & TCTG & NPM1 & L287fs & 40 & 0 & nd & YES & 698 & 36.5 \\
\hline $340 P$ & chr13 & 28608244 & 28608244 & - & $1^{\star}$ & FLT3 & $2^{\star}$ & nd & 0 & nd & YES & 10884 & 1.9 \\
\hline $340 P$ & chr17 & 7577548 & 7577548 & C & $\mathrm{T}$ & TP53 & G206S & 61.9 & 0 & 0 & YES & 5151 & 0.6 \\
\hline $340 \mathrm{P}$ & chr11 & 32417914 & 32417914 & - & $\mathrm{T}$ & WT1 & R380fs & 50.53 & 0 & 32.14 & YES & 6096 & 38.9 \\
\hline $584 \mathrm{P}$ & chr5 & 170837543 & 170837543 & - & TCTG & NPM1 & L287fs & nd & 0 & nd & YES & 553 & 40.3 \\
\hline $584 \mathrm{P}$ & chr2 & 25457252 & 25457252 & $\mathrm{~T}$ & C & DNMT3A & N879D & 20 & 37 & 47.62 & YES & 1133 & 62.5 \\
\hline $584 \mathrm{P}$ & chr2 & 209113112 & 209113112 & C & $\mathrm{T}$ & IDH1 & $\mathrm{R} 132 \mathrm{H}$ & 31.94 & 0 & 0 & NO & 3550 & 0 \\
\hline $584 \mathrm{P}$ & chr4 & 55599321 & 55599321 & A & $\mathrm{T}$ & KIT & D816V & 30.9 & 0 & 0 & NO & 16031 & 0 \\
\hline $1019 P$ & chr5 & 170837545 & 170837545 & & & NPM1 & L287fs & nd & 0 & nd & YES & 423 & 21.7 \\
\hline $1019 \mathrm{P}$ & chr2 & 25457242 & 25457242 & C & $T$ & DNMT3A & $\mathrm{R} 882 \mathrm{H}$ & 68.75 & 0 & 66.67 & YES & 862 & 49.4 \\
\hline $1019 \mathrm{P}$ & chr2 & 209113112 & 209113112 & C & $\mathrm{T}$ & IDH1 & $\mathrm{R} 132 \mathrm{H}$ & 45.45 & 0 & 0 & YES & 4553 & 0.2 \\
\hline
\end{tabular}

suggesting clearance of the initial leukemic clone and occurrence of a novel leukemia at the time of relapse.

On the contrary, in all $N P M 1^{\text {mut }}$ persistent pts at least three common mutations (mean 7.6) between diagnosis and relapse were found (Fig. 3a and Supplementary Data 2). Furthermore, preleukemic mutations persisted in only six of the 10 pts during remission, and in these cases the VAF was lower compared to $N P M 1^{\text {mut }}$ loss pts (Supplementary Fig. 2b). These findings suggest that the preleukemic clone is not yet fully dominating hematopoiesis in all NPM1 ${ }^{\text {mut }}$ persistent pts. However, reappearance of preleukemic mutations in conjunction with the persistence of $N P M 1^{\text {mut }}$ and other mutations at the time of relapse suggests that a leukemic stem cell clone survived initial chemotherapy and gave rise to relapsed disease shaped by clonal evolution.

We performed deep amplicon sequencing to survey diagnosis samples from three $N P M 1^{\text {mut }}$ loss and three $N P M 1^{\text {mut }}$ persistent pts for relapse specific mutations at the time of diagnosis. First, we wanted to see whether relapse specific mutations in NPM1 $1^{\text {mut }}$ persistent cases were already present at diagnosis, and second we wanted to rule out the possibility that a minor $N P M 1^{\text {mut }}$ loss subclone was already present at this time point. Coverage for the respective variant positions ranged from 423 to 16031 reads (mean 4224, median 3023). In none of the $N P M 1^{\text {mut }}$ loss diagnosis samples we were able to detect a relapse specific mutation (Table 1) while in $N P M 1^{\text {mut }}$ persistent pts we detected a relapse specific mutation in 2 out of 3 pts with VAFs of 0.6 and $0.17 \%$, respectively (TP53 $3^{\text {mut }}$ in ID340 and $I D H 1^{\text {mut }}$ in ID1019).

To explore whether mutated genes perturb similar biological pathways in diagnosis and relapse samples, we performed gene set over representation analysis in $N P M 1^{\text {mut }}$ loss and $N P M 1^{\text {mut }}$ persistent pts at both time points. We found in both $N P M 1^{\text {mut }}$ loss as well as NPM1 ${ }^{\text {mut }}$ persistent cases diagnostic mutations to be enriched for members of the ERBB and MYC signaling pathways (Fig. 3b and Supplementary Data 3). For NPM1 mut persistent cases diagnosis and relapse associated mutations showed a highly concordant mutational pattern with mutations being enriched for members of the MYC, SCF-KIT (stem cell factor) and WNT signaling pathways. In contrast, the mutational spectrum of $N P M 1^{\text {mut }}$ loss relapse samples affected very different pathways, such as MAPK signaling and pathways known to be play a role in cancer (Pathways in Cancer), although a more stem cell-like phenotype (WNT signaling) was seen in these cases (Fig. 3b).
As previous chemotherapy might affect cancer mutation signatures, i.e. the type of single nucleotide variants (SNVs), we assessed the transversion frequency of mutations found in diagnosis and relapse samples from $N P M 1^{\text {mut }}$ loss and $N P M 1^{\text {mut }}$ persistent pts to evaluate whether $N P M 1^{\text {mut }}$ loss at relapse might be considered as tAML following cytotoxic therapy of the initial de novo AML. While Wong et al. ${ }^{18}$ recently investigated 22 pts with tAML and found no difference in transversion frequency compared to de novo AML and secondary AML (sAML), Ding et al. ${ }^{17}$ reported a strong increase of transversions after chemotherapy in relapse compared to primary tumors. Here, we saw an increase of mainly $\mathrm{C}$ to $\mathrm{A}$ transversions from diagnosis to relapse in both groups (Fig. $4 \mathrm{a}),\left(N P M 1^{\text {mut }}\right.$ loss: $p=0.023$; $N P M 1^{\text {mut }}$ persistent: $p=0.014$, two-tailed Mann-Whitney test, Fig. 4b).

RNA-Seq of NPM1 ${ }^{\text {mut }}$ loss and persistent pts. To determine whether specific mutational patterns in diagnosis and relapse samples of NPM1 ${ }^{\text {mut }}$ loss pts have an impact on gene expression, we performed RNA-Seq. The expression of 28,922 genes above detectable expression levels ( $>1$ read in at least one sample) was compared in a pairwise approach between diagnosis $(n=5)$ and relapse $(n=5)$ NPM1 $1^{\text {mut }}$ loss samples. This resulted in 2141 differentially expressed genes $(p \leq 0.05)$ (Fig. 5a and Supplementary Data 4). Among these, we identified HOXA10, HOXB6, and MEIS1, an NPM1 ${ }^{\text {mut }}$ associated homeobox (HOX) expression signature ${ }^{19}$, highly expressed in diagnosis samples, which was not seen in $N P M 1^{\text {mut }}$ loss relapse samples.

Next, we used the $N P M 1^{\text {mut }}$ signature from Verhaak and colleagues $^{19}$, comprising a total of 18 genes, which can discriminate between $N P M 1^{\text {mut }}$ and $N P M 1$ wildtype $\left(N P M 1^{\mathrm{wt}}\right)$ $A M L$, for hierarchical clustering of paired diagnosis and relapse samples from $5 N P M 1^{\text {mut }}$ loss and $5 N P M 1^{\text {mut }}$ persistent pts. This $N P M 1^{\text {mut }}$ signature grouped the relapse samples of the five cases that had lost the NPM1 ${ }^{\text {mut }}$ at relapse (NPM1 $1^{\text {wt }}$ cases) next to the $15 N P M 1^{\text {mut }}$ samples. This $N P M 1^{\text {wt }}$ group is characterized by high expression of $A P P, N R G N$ and low expression of $H O X B 5$, HOXB6, HOXA9 and HOXA10 (Supplementary Fig. 3).

Gene set enrichment analysis (GSEA) further supported our genomic WES based findings, as the gene expression signature of NPM1 $1^{\text {mut }}$ loss relapse samples showed an enrichment of genes 

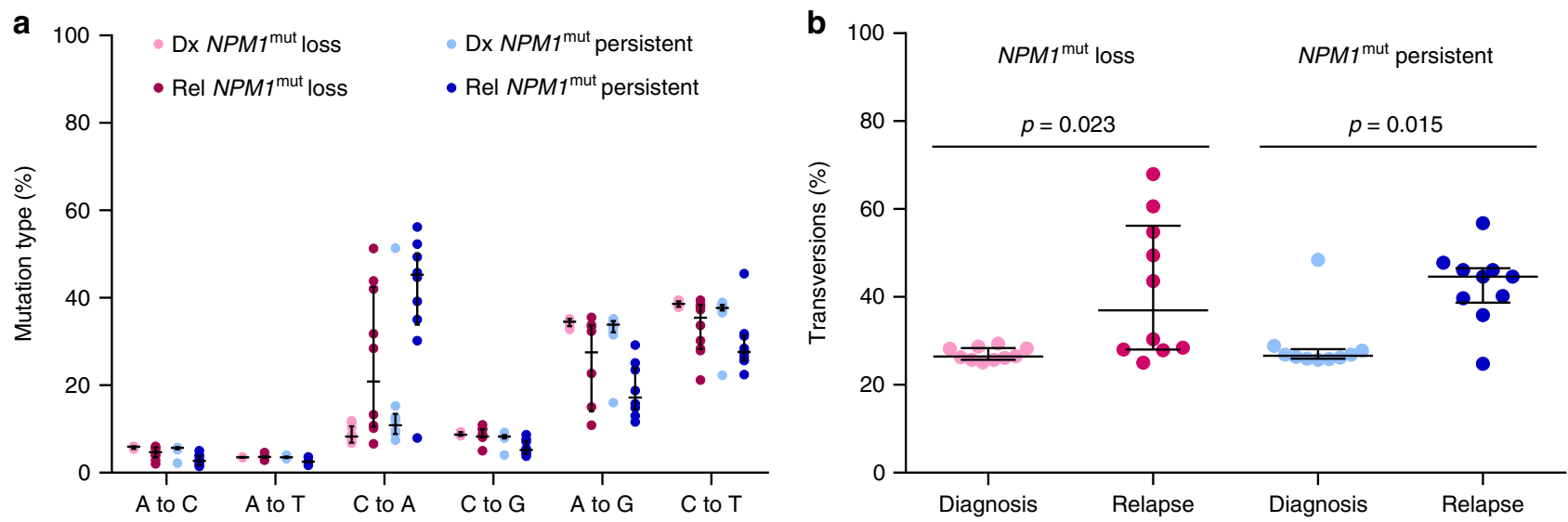

Fig. 4 Mutation types before and after chemotherapy in NPM $7^{m u t}$ loss and persistent pts. a Frequency of transversions ( $A$ to $C, A$ to $T, C$ to $A$, and $C$ to $G$ ) and transitions ( $\mathrm{A}$ to $\mathrm{G}$ and $\mathrm{C}$ to $\mathrm{T}$ ). b Proportion of transversions in NPM7mut loss diagnosis (rose) and relapse (pink) samples and NPM7mut persistent diagnosis (light blue) and relapse (dark blue) samples
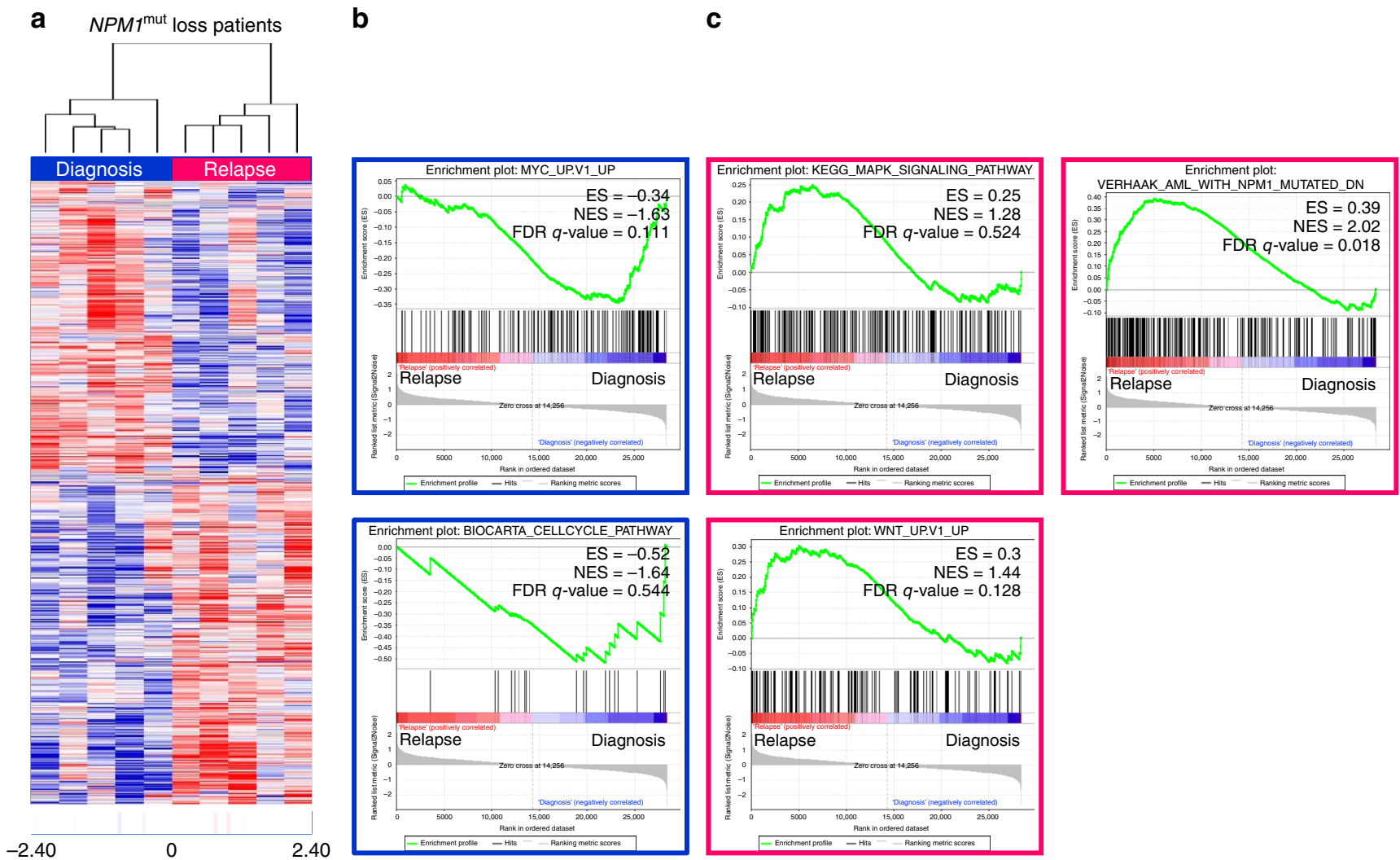

Fig. 5 Heatmap of differentially expressed genes and GSEA of diagnosis versus relapse NPM $7^{\text {mut }}$ loss samples. a Differentially expressed genes (significant at 0.05 level of the univariate test) from RNA-Seq were assessed using the ClassComparison option from BRB-ArrayTools. Blue indicates upregulation and red downregulation between groups. b Diagnosis samples show enrichment of MYC_UP.V1_UP and BIOCARTA_CELLCYCLE_PATHWAY gene sets. c Relapse samples were enriched in KEGG_MAPK_SIGNALING_PATHWAY, WNT_UP.V1_UP gene sets and genes which are downregulated in NPM7mut samples (VERHAAK_AML_WITH_NPM1_MUTATED_DN). ES, enrichment score; FDR, false discovery rate; NES, normalized enrichment score

belonging to Pathways in cancer and more specifically of MAPK and WNT signaling, while in diagnosis samples pathways related to cell cycle and MYC signaling were affected (Fig. 5b, c and Supplementary Data 5). In addition, in relapse samples we found a significant upregulation of genes, which have been shown to be down-regulated in $N P M 1^{\text {mut }}$ AML (FDR $q$-value $\left.=0.018\right)(\text { Fig. } 5 \text { c })^{19}$. This demonstrates that relapse samples are $N P M 1^{\mathrm{wt}}$ also on the functional level.

CTNNB1 levels in NPM1 ${ }^{\text {mut }}$ diagnosis samples. To confirm stronger $\mathrm{WNT}$ signaling in $N P M 1^{\text {mut }}$ persistent diagnosis samples compared to NPM1 ${ }^{\text {mut }}$ loss diagnosis samples, which was predicted by pathway analysis of mutated genes and transcriptomic analysis, we performed flow cytometry in three diagnosis samples of NPM1 $1^{\text {mut }}$ loss and NPM1 mut persistent cases, respectively. Using an intracellular staining protocol for CTNNB1 (ß-Catenin), which is a key player in the WNT signaling pathway and crucial for leukemic stem cells ${ }^{20}$, we could confirm higher levels of both total CTNNB1 and active CTNNB1 in NPM1 $1^{\text {mut }}$ persistent diagnosis samples compared to $N P M 1^{\text {mut }}$ loss diagnosis samples ( $p=0.04$, unpaired $t$-test; Fig. 6). 
a

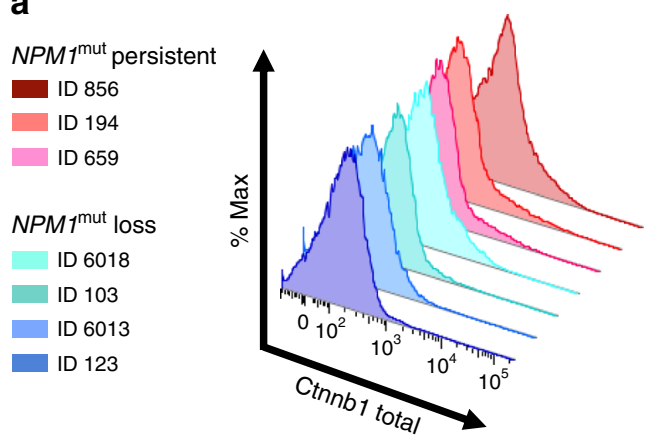

b

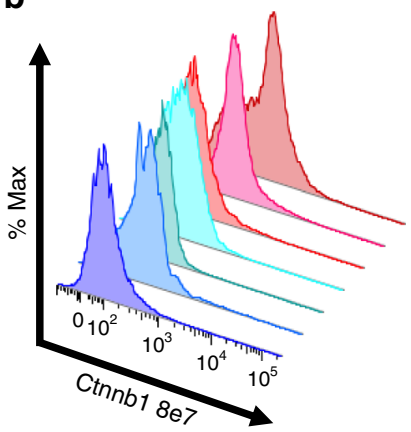

C

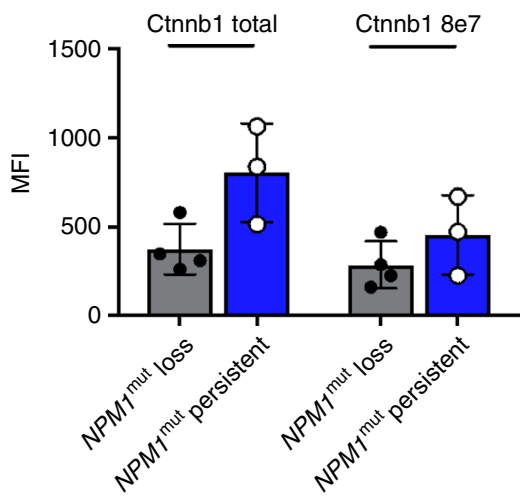

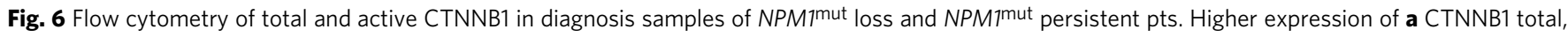
b CTNNB1 $8 \mathrm{e} 7$ in 3 NPM7mut persistent diagnosis samples compared to 4 NPM1mut loss diagnosis samples, and c mean fluorescent intensity (MFI) including all data points, data is presented as mean \pm s.d

NPM1 $1^{\text {mut }}$ loss is characterized by a distinct clinical course. As the mutational pattern pointed to a distinct biology in pts with $N P M 1^{\text {mut }}$ loss, we were interested to see whether these pts differ with regard to their clinical outcome. Indeed, $N P M 1^{\text {mut }}$ loss pts had a significantly longer remission duration compared to pts with persisting $N P M 1^{\text {mut }}$. Median time to relapse in the $11 \mathrm{NPM} 1^{\text {mut }}$ loss pts was 30 months [95\% Confidence Interval (CI), 21 months -not reached] compared to 8 months in 95 pts maintaining the $N P M 1^{\text {mut }}$ at relapse (95\% CI, 7 months -10 months; $p=0.0004$, two-sided log-rank test; Fig. 7). In line with our previous report ${ }^{7}$, $N P M 1^{\text {mut }}$ loss pts poorly responded to salvage chemotherapy; here only 2 of 11 pts achieved a second complete remission (CR) whereas 58 of $96(60 \%) N P M 1^{\text {mut }}$ persistent pts with clinical data available achieved a second CR ( $p=0.008$, Fisher's exact test).

Possible mechanisms of relapse in NPM1 ${ }^{\text {mut }}$ AML. Based on the mutation profile, the gene expression signature, and also the clinical course of $N P M 1^{\text {mut }}$ loss and NPM1 ${ }^{\text {mut }}$ persistent pts, two different mechanisms of relapsed disease can be discussed (Fig. 8). In $N P M 1^{\text {mut }}$ persistent pts an NPM1 $1^{\text {mut }}$ leukemic clone outlasts chemotherapy, undergoes additional evolution and subsequently sprouts to cause the relapse. In contrast, in $N P M 1^{\text {mut }}$ loss pts the initial leukemic clone is eradicated by chemotherapy. However, dominant clonal hematopoiesis with persistence of preleukemic mutations, such as DNMT3A $A^{\text {mut }}$, provides the basis for the development of a second AML, caused by a transforming event different from NPM1 ${ }^{\text {mut }}$ and thereby leading to a novel leukemia rather than to a relapse of the initial leukemia. This is further supported by the significantly longer remission duration in the $N P M 1^{\text {mut }}$ loss pts. In accordance, evaluation of VAF on average depicts persistence of preleukemic mutations in $N P M 1^{\text {mut }}$ loss pts, with an unchanged VAF at remission as shown in Fig. 8 (see also Supplementary Fig. 4). In contrast, in $N P M 1^{\text {mut }}$ persistent pts we frequently observe clearance of the leukemic clone at remission below the detection limit of our WES approach and recurrence of the mutations become apparent at relapse (Fig. 8 and Supplementary Fig. 4).

\section{Discussion}

With regard to clonal evolution of $N P M 1^{\text {mut }}$ cases at the time of relapse, this extended study of $129 \mathrm{NPM} 1^{\text {mut }}$ pts confirmed our previous findings performed in a smaller cohort of $N P M 1^{\text {mut }} \mathrm{pts}^{7}$ but also provides novel insights into relapse pathomechanisms of $N P M 1^{\text {mut }}$ AML. In general, $N P M 1^{\text {mut }}$ persistence was the predominant relapse mechanism of $N P M 1^{\text {mut }}$ AML, since we detected persisting $N P M 1^{\text {mut }}$ clones at relapse in $91 \%$ of pts.

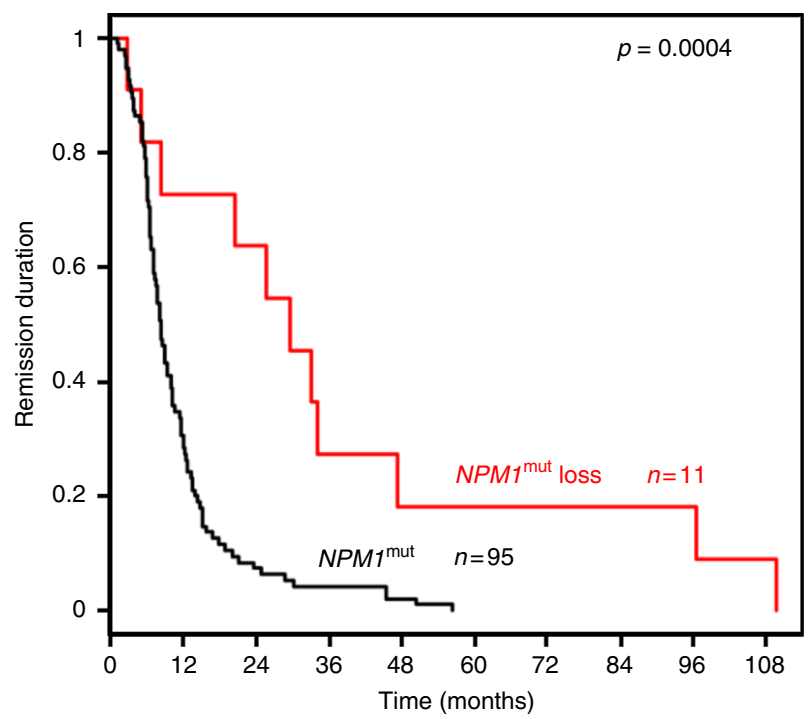

Fig. 7 Remission duration of NPM1mut loss and persistent pts. Pts with NPM1mut loss (red) had a significantly longer remission duration compared to pts maintaining NPM1mut (black) at relapse

Moreover, detectable $N P M 1^{\text {mut }}$ mutation after morphological remission was accompanied by a morphological relapse, illustrating the necessity of monitoring minimal residual disease (MRD) in NPM1 ${ }^{\text {mut }}$ pts. By SNP analysis we could confirm critical regions associated with disease relapse such as deletion of 12 p13 leading to haploinsufficiency of ETV6, a tumor suppressor gene also frequently lost in AML with complex karyotypic changes $^{21}$. Similarly, loss of $17 \mathrm{q} 11.2$ leads to NF1 haploinsufficiency, which was previously shown to contribute synergistically to disease progression ${ }^{22}$. Relapse specific gain of $11 \mathrm{q} 23.3$ affects the oncogene KMT2A (MLL) whereas the mechanism of trisomy 8 still remains elusive. The frequently acquired UPD of chromosome 13 results in homozygous FLT3-ITD mutations. As shown in our previous study only few genomic aberrations were present in NPM1 ${ }^{\text {mut }}$ diagnosis samples (0.3/patient), whereas at the time of relapse we observed a substantial increase (1/patient) in the number of genomic alterations.

Comparative mutation profiling confirmed the highest stability of DNMT3A $A^{\text {mut }}(95 \%)$, due to the early acquisition and preleukemic occurrence ${ }^{11}$. Similarly, other mutations associated with clonal hematopoiesis, such as $I D H 1$ and $I D H 2$ mutations, also showed a high stability ( 86 and $88 \%$, respectively). These genes 

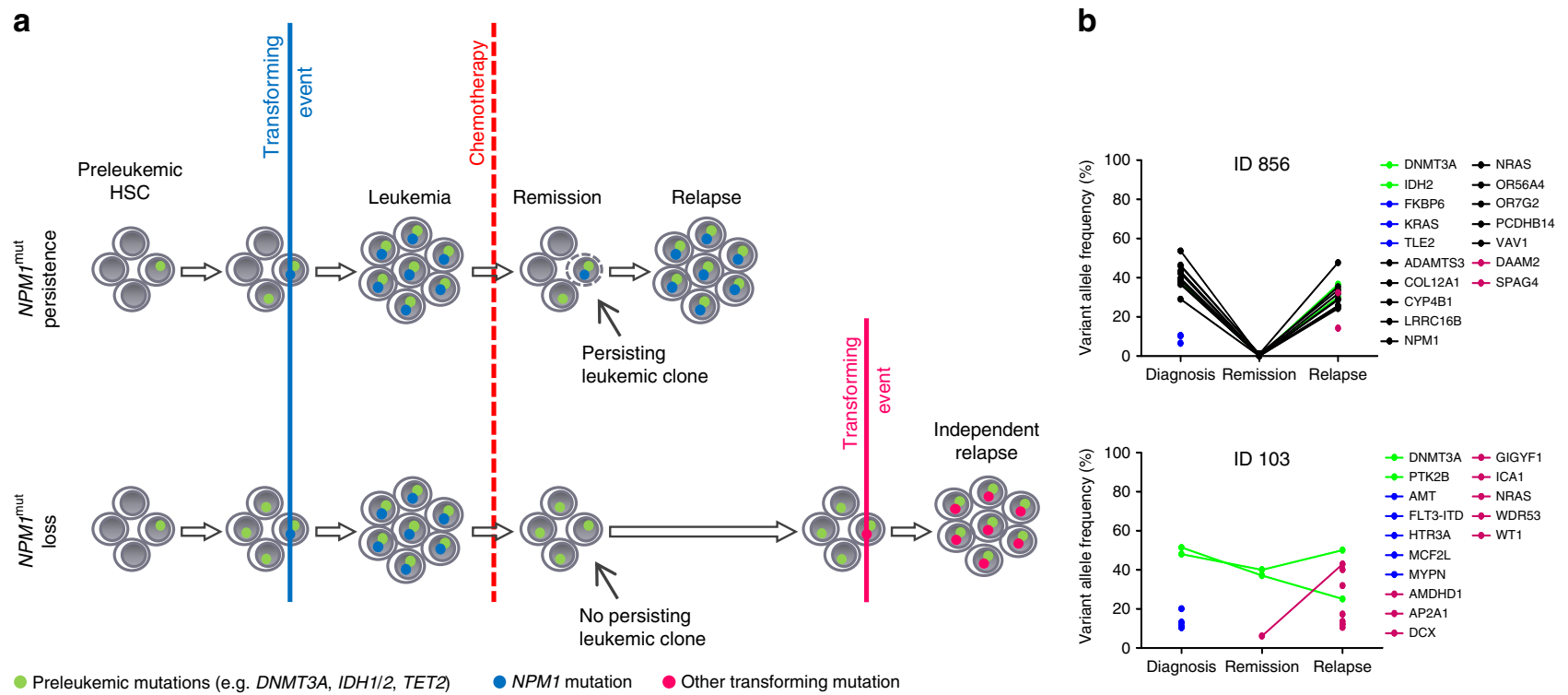

Fig. 8 Possible mechanisms of relapse in NPM7mut $A M L$. a Based on our mutation data we postulate different mechanisms of relapse for NPM ${ }^{m u t}$ loss and $N P M 7^{m u t}$ persistent pts. $\mathbf{b}$ Variant allele frequency plots of mutated genes from exemplary pts for each group

are involved in epigenetic regulation and mutations result in a preleukemic state of $\mathrm{HSCs}^{5,10}$. Preleukemic mutations confer an advantage in self-renewal and proliferation of HSCs, thereby leading to clonal expansion ${ }^{23,24}$. Moreover, it has been shown that DNMT3A ${ }^{\text {mut }}$ HSCs can survive induction therapy and persist at remission, thus supposedly increasing the risk for a second $\mathrm{AML}^{11,25}$. NPM1 ${ }^{\text {mut }}$ was also a relatively stable marker, yet $9 \%$ of pts were NPM1 ${ }^{\text {wt }}$ at relapse. In contrast NRAS and FLT3 mutations were less stable, because these driver mutations are rather late events, just like other mutations in genes involved in active signaling ${ }^{5,6}$.

Interestingly, WES detected PTK2B and PAX5 mutations persisting at the time of remission in two NPM1 ${ }^{\text {mut }}$ loss pts. These mutations have not been previously described as preleukemic or germline mutations. PTK2B is a tyrosine kinase involved in the PI3K-AKT signaling pathway. PAX5 is a transcription factor, and encodes the $\mathrm{B}$ cell lineage specific activator protein. In another $N P M 1^{\text {mut }}$ loss case a mutation in BCOR, a transcriptional corepressor that interacts with histone deacetylases, hence another epigenetic modifier, was identified as preleukemic mutation. $B C O R L 1$, a paralog of $B C O R$, was found recurrently mutated in individuals without hematological malignancy ${ }^{24}$, and $B C O R$ mutations have recently been linked to clonal hematopoiesis in aplastic anemia ${ }^{26}$ as well as secondary AML and tAML ${ }^{27}$. An additional potential preleukemic mutation, $N P A T^{\text {mut }}$, which persisted in remission, was found in an $N P M 1^{\text {mut }}$ persistent patient. NPAT is found mutated in several cancer types including lung cancer ${ }^{28}$ and malignant melanoma ${ }^{29}$, and germline NPATmut was recently identified as a candidate risk factor for Hodgkin lymphoma ${ }^{30}$, thereby suggesting also a possible implication in AML pathogenesis.

Thus, except for preleukemic mutations the mutational profiles of $N P M 1^{\text {mut }}$ loss pts showed almost no overlap between diagnosis and relapse samples. This observation points to a significant difference in leukemia biology, which is also reflected by the fact that diagnosis and relapse associated gene mutations targeted different pathways. Notably, the diagnosis mutational pattern of $N P M 1^{\text {mut }}$ loss pts was similar to cases with $N P M 1^{\text {mut }}$ persistence, which further confirms $N P M 1^{\text {mut }}$ as disease-defining event ${ }^{31}$. Furthermore, it shows that $N P M 1^{\text {mut }}$ associated pathomechanisms lead to distinct cooperating events. In line, diagnosis and relapse mutational patterns of $N P M 1^{\text {mut }}$ persistent pts were also closely related and formed a cluster together with $N P M 1^{\text {mut }}$ loss diagnosis samples, which was separate to the ' $N P M 1^{\text {wt' }}$ samples when clustering the NPM1 associated molecular signature.

This suggests that at diagnosis $N P M 1^{\text {mut }}$ was the disease transforming event in both groups. In persistent pts, chemotherapy might lead to the repression and possibly elimination of the major leukemic clone, which at the time of remission cannot be detected anymore at the sensitivity level of our sequencing analysis. However, in these cases there is persistence of leukemic subclones that undergo clonal evolution and cause leukemia relapse. In $N P M 1^{\text {mut }}$ loss cases, chemotherapy might lead to the extinction of the leukemic clones, but persistent clonal hematopoiesis, which is dominating over healthy hematopoiesis in these cases, increases the risk for a second "de novo" AML. This hypothesis is underlined by the different mutation profiles as well as the longer duration until disease recurrence in these cases (median, 30 months vs. 8 months) since acquisition of a new driver mutation in this pre-leukemic clonal hematopoiesis setting is likely to take longer than a relapse originating from a persisting leukemic subclone. This clinical finding was recently confirmed by Höllein and colleagues ${ }^{32}$.

Due to limitations of WES in detecting rare variants, we conducted deep targeted sequencing of selected diagnosis samples to search for mutations in minor subclones which are present with a VAF down to $0.5 \%$. We did not discover a relapse specific mutation at this VAF in any of the NPM1 ${ }^{\text {mut }}$ loss samples, which suggests that all leukemic clones are eradicated. Moreover, the analysis of the types of point mutations revealed a higher average percentage of transversions at the time of relapse in $N P M 1^{\text {mut }}$ persistent compared to loss samples (45\% versus $27 \%)$. This higher number of transversions in persistent pts is comparable with data reported by Ding and colleagues ${ }^{17}$ who also found an increase of transversions in relapse samples.

A potential explanation, why in some $N P M 1^{\text {mut }}$ pts the disease might be initially cured and the relapse then occurs as an independent event based on persisting clonal hematopoiesis can be derived from our gene set analyses. Interestingly, diagnostic $N P M 1^{\text {mut }}$ loss samples showed less stem cell-like phenotypic features due to absent enrichment of mutations in the WNT signaling pathway, a hallmark for leukemic stem cells, which acts 
as a regulator of self-renewal ${ }^{20}$. In contrast, $N P M 1^{\text {mut }}$ leukemias harboring more stem cell features might be more resistant to chemotherapy and thus they will eventually relapse, which in turn is characterized by the persistence of the $N P M 1^{\text {mut }}$. Indeed, increased WNT signaling, reflected by higher CTNNB1 levels, in $N P M 1^{\text {mut }}$ persistent diagnosis samples compared to $N P M 1^{\text {mut }}$ loss diagnosis samples was confirmed by flow cytometry analysis.

In $N P M 1^{\text {mut }}$ loss pts, over time there might be a selection and/ or clonal evolution of a more aggressive DNMT3A mut HSC, which results in a relapse AML also characterized by stem celllike features. As in the general population with presence of clonal hematopoiesis this transformation process is slow and likely requires the acquisition of additional hits. Loss of $N P M 1^{\text {mut }}$ is a rare event and thus monitoring $N P M 1^{\text {mut }}$ minimal residual disease is nevertheless a valid marker for relapse prediction ${ }^{33,34}$. In addition, our observation further highlights the need to develop drugs which target these epigenetic regulator mutations to eradicate clonal hematopoiesis as precancerous/preleukemic lesion ${ }^{35}$, and even more importantly it shows the necessity to reveal the mechanism and role of preleukemic mutations.

In summary, we could show that relapsed AML of $N P M 1^{\text {mut }}$ loss pts possesses almost no relationship to the primary tumor so that a more suitable nomenclature would be second "de novo" AML. Further investigations are needed to evaluate whether this phenomenon can also be seen in relapses which have lost other distinct driver lesions, such as $C E B P A^{\text {mut }}, \mathrm{t}(8 ; 21)$ or $\operatorname{inv}(16)$. Second "de novo" AML, due to persistence of preleukemic mutations, has strong implications for the clinic since MRD monitoring has limited value in these pts. Moreover, in cases with dominant clonal hematopoiesis at the time of remission, novel treatment approaches are needed to eradicate preleukemic clones. Finally, the development of more sensitive MRD tools to detect persisting leukemic subclones will contribute to ultimately achieve cure and prevent relapse in AML pts.

\section{Methods}

Patients. In total, $129 \mathrm{NPM} 1^{\text {mut }}$ AML pts (25-78 years) with availability of samples at least at the time of diagnosis and at relapse were analyzed. $84 \%$ of the pts $(109 / 129)$ were treated in one of four prospective AMLSG treatment trials [HD98A $(n=9$; NCT00146120), AMLSG 07-04 $(n=34$; NCT00151242), AMLSG 09-09 ( $n=64$; NCT00893399) and AMLSG 16-10 $(n=2$; NCT01477606)]; the remaining 20/129 pts (16\%) participated in the AMLSG BiO Registry study (NTC 01252485). All pts received intensive standard induction chemotherapy with cytarabine and an anthracycline $(7+3$ regimen $)$ followed by high-dose cytarabine consolidation cycles with few exceptions in $N P M 1^{\text {mut }}$ persistence pts. Patient characteristics are summarized in Table 2. Informed consent for both treatment and biobanking of leukemia samples according to the Declaration of Helsinki was given by all pts. Approval was obtained from the ethical review board of the University of Ulm (ethical vote number 148/10).

Mutation analysis. NPM1 $1^{\text {mut }}$ was diagnosed by locked nucleic acid (LNA)-based $\mathrm{PCR}^{36}$, DNMT3A mutation screening was performed by DNA-based amplification of the corresponding exons ${ }^{37}$. Subsequently, amplified DNA was used for mutational analysis by conventional Sanger sequencing to define the exact mutation types. FLT3-ITD, FLT3-TKD and ASXL1 amplicons were screened for mutations by a GeneScan-based fragment analysis ${ }^{38,39}$. The amplified fragments of IDH1, IDH2, TP53, and NRAS were analyzed by denaturing high-performance liquid chromatography (DHPLC) on a WAVE 3500HT DNA Fragment Analysis System (Transgenomic) ${ }^{40-42}$. Samples classified as mutated were further characterized by direct sequencing. $M L L$-PTD was detected by RNA-based $\mathrm{PCR}^{43}$.

Single nucleotide polymorphism microarray analysis. A total of $500 \mathrm{ng}$ of purified high-quality genomic DNA (using the QIAamp DNA Mini Kit, Qiagen) from every sample was digested, ligated to adaptors, amplified by PCR, purified, fragmented, labeled and hybridized to the Genome-Wide Human SNP Array 6.0 according to manufacturer's instructions (Affymetrix). CEL files were generated using the Command Console Software 4.1.2 (Affymetrix). The Genotyping Console Software 2.0 (Affymetrix) was used for normalization and analysis of CEL files.

Whole-exome sequencing analysis. WES was performed on diagnosis, remission and relapse samples from 10 pts with $N P M 1^{\text {mut }}$ loss and 10 pts with $N P M 1^{\text {mut }}$

\section{Table 2 Clinical characteristics of 129 NPM1mut patients at diagnosis}

\section{Variable}

No.

Age, years

Median

Range

25-78

Sex

Female

Male

AML history

De novo

tAML

Not available

Cytogenetics

$\mathrm{CN}$

Deletion 9q

Others

Not available

$\begin{array}{ll} & 54 \\ & 25-78 \\ & \end{array}$

NPM1 mutation type

A

B

12

Others

65

108

2

19

102

5

3

19

WBC count, $\times 10^{9} / L$

Median

Range

BM Blasts, \%

Median

Range

100

12
9

8

\section{5}

Induction cycles, $n$

1

2

Remission status after first induction cycle $(n=129)$

$\begin{array}{ll}\text { CR/CRi } & 97 \\ \text { PR } & 31 \\ \text { RD } & 1\end{array}$

Remission status after second induction cycle $(n=113)$

CR

112

Relapse

Consolidation therapy $(n=127)$

High-dose Cytarabine

Allogeneic SCT

Autologous SCT

Overall survival, days

Median

1

97

31

114

9

4

\section{2}

$C N$ cytogenetically normal, WBC white blood cell, $B M$ bone marrow, $C R$ complete remission, $C R i$ complete remission with incomplete hematologic recovery, $P R$ partial remission, $R D$ refractory disease, SCT stem cell transplantation 
remission sample were assessed individually for authentic contribution to disease. Gene set analysis was performed to compute overlap between the mutated genes of each group (NPM1 ${ }^{\text {mut }}$ loss diagnosis, $N P M 1^{\text {mut }}$ loss relapse, $N P M 1^{\text {mut }}$ persistent diagnosis and $N P M 1^{\text {mut }}$ persistent relapse) with gene sets from the Molecular Signatures Database (MSigDB, http://software.broadinstitute.org/gsea/msigdb/ annotate.jsp $)^{51}$. We selected chemical and genetic perturbations, canonical pathways, BioCarta gene sets, KEGG gene sets, Reactome gene sets, microRNA targets, transcription factor targets, and oncogenic signatures gene sets with the FDR $q$-value threshold set to 0.05 . For calculation of SNV frequencies from the different mutation classes, mutations below $20 \% \mathrm{VAF}$ were filtered out to reduce the number of false positives. Statistical significance was assessed using a nonparametric Mann-Whitney test.

Deep amplicon sequencing. A custom amplicon panel was used to sequence selected relapse specific mutations in three $N P M 1^{\text {mut }}$ loss and three $N P M 1^{\text {mut }}$ persistent diagnosis samples with deep coverage. We used an AML custom design amplicon panel which includes 31 genes most relevant in the oncogenesis of AML. We selected six diagnosis samples to cover as many relapse specific mutations as possible with our panel. Target enrichment was performed from $50 \mathrm{ng}$ DNA using the HaloPlex HS Target Enrichment System (Agilent) according to the manufacturer's instructions. Pooled DNA libraries were sequenced on an Illumina MiSeq with the MiSeq Reagent Kit v2 (300-cycles, Illumina). One bp was trimmed from all reads, which were then aligned to the human reference genome GRCh37 using BWA-MEM ${ }^{44}$. Molecular barcodes were deduplicated using BamDeduplicateByBarcode from ngs-bits ${ }^{52}$ and alignments were sorted and indexed using Picard (Picard 1.138, http://broadinstitute.github.io/picard) and locally realigned with GATK (Indel Realigner, GATK 3.4-46) ${ }^{46}$. Resulting alignments were used for coverage calculation with BEDTools ${ }^{53}$ as well as for pileup generation and variant calling with SAMtools ${ }^{54}$ and VarScan $2^{45}$ respectively. BAM files were inspected with the integrative Genomics Viewer (Broad Institute, Cambridge, MA) for presence of relapse specific mutations. HaloPlexHS is an improved next-generation targeted enrichment method allowing tracking and correction of PCR duplicates by tagging every single original DNA molecule with a molecular barcode. Using the HaloPlexHS protocol it is possible to detect rare variants down to $0.5 \% \mathrm{VAF}^{55}$.

RNA-Seq analysis. RNA was extracted from paired diagnosis/relapse samples of five pts with $N P M 1^{\text {mut }}$ loss and five pts with $N P M 1^{\text {mut }}$ persistence using the AllPrep DNA/RNA Mini Kit (QIAGEN), and RNA quality was assessed using a BioAnalyzer 2100 (Agilent). Libraries were prepared from $1 \mu \mathrm{g}$ of total RNA using the TruSeq Stranded Total RNA Kit with Ribo-Zero Human/Mouse/Rat (Illumina) according to the manufacturer's instructions. The pooled RNA libraries were sequenced on an Illumina HiSeq2000 to obtain 100 bp paired-end reads. RNA-Seq reads were aligned to the human reference genome hg 19 and quantified using STAR v.2.4.2 $\mathrm{a}^{56}$ in the 2-pass mapping mode. Furthermore, the DESeq $2^{57}$ package from $\mathrm{R}$ was used to obtain normalized expression values. BRB-ArrayTools Version 4.5.0 Beta_ $2^{58}$ (BRB, National Cancer Institute, Bethesda, MD, USA) and GSEA (http://broadinstitute.org/gsea/index.jsp) ${ }^{51}$ were used for class comparison analyses. Hierarchical clustering and heatmap visualization of differentially expressed genes was performed using Cluster $3.0^{59}$ and Java Treeview ${ }^{60}$.

Flow cytometry. Intracellular flow cytometry was performed using the Fix \& Perm Cell Permeabilization Kit (Invitrogen) ${ }^{61}$. In brief, primary patient cells were resuspended in $100 \mu \mathrm{l}$ Fixation Medium and incubated for $15 \mathrm{~min}$ at room temperature. Cells were then washed in $3 \mathrm{ml} \mathrm{PBS}+0.1 \% \mathrm{NaN}_{3}+5 \%$ FBS followed by centrifugation. Staining with the respective antibodies and isotope controls was performed in $100 \mu \mathrm{l}$ of the permeabilization medium according to the manufacturer's instructions for $1 \mathrm{~h}$ at room temperature followed by washing and centrifugation as indicated above. We used a primary antibody against total CTNNB1 (anti-h-beta-Catenin, APC-conjugated, \#IC13292A, R\&D Systems) or active CTNNB1 (anti-active-b-Catenin, clone 8E7, \#05-665-25UG, Merck Millipore) followed by a secondary Alexa488 labeled antibody (donkey anti-mouse, Invitrogen \# R37114). Cells were analyzed using a CantoII ${ }^{\mathrm{TM}}$ (Becton-Dickinson) cytometer. Data analysis was performed using FlowJo ${ }^{\mathrm{TM}}$ software (Treestar, Ashland, OR).

Statistical analyses. Statistical analyses for clinical outcome were performed as follows: The definition of CR, remission duration and overall survival (OS) were based on recommended criteria from the 2017 ELN recommendations ${ }^{4}$. Remission duration endpoints measured from the date of documented CR were relapse (failure), death in CR (censored) and alive in CR at last follow-up (censored). The Kaplan-Meier method was used to estimate distributions of remission duration and differences between two groups were analyzed using the two-sided log-rank test. Response to salvage therapy was analyzed using Fisher's exact test and expression data with normal distribution by unpaired t-test. An effect was considered significant if $P \leq 0.05$. All statistical analyses were performed with the statistical software environment $\mathrm{R}$ version 3.0.2, using the $\mathrm{R}$ packages Hmisc version $3.13-0$ and survival version $2.37-7^{62}$.

Reporting summary. Further information on research design is available in the Nature Research Reporting Summary linked to this article.

\section{Data availability}

SNP microarray data and RNA-Seq data that support the findings of this study have been deposited in the NCBI Gene Expression Omnibus ${ }^{63}$ (GEO) and are accessible through GEO Series accession numbers GSE46951 and GSE128604. WES raw data that support the findings of this study are available from the corresponding author upon reasonable request. Evaluated true variants from WES and deep amplicon sequencing are provided within the paper and its supplementary information files.

Received: 4 March 2018 Accepted: 28 March 2019

Published online: 02 May 2019

\section{References}

1. Döhner, H., Weisdorf, D. J. \& Bloomfield, C. D. Acute myeloid leukemia. N. Engl. J. Med. 373, 1136-1152 (2015).

2. Arber, D. A. et al. The 2016 revision to the World Health Organization classification of myeloid neoplasms and acute leukemia. Blood 127, 2391-2405 (2016).

3. Döhner, H. et al. Diagnosis and management of acute myeloid leukemia in adults: recommendations from an international expert panel, on behalf of the European LeukemiaNet. Blood 115, 453-474 (2010).

4. Döhner, H. et al. Diagnosis and management of AML in adults: 2017 ELN recommendations from an international expert panel. Blood 129, 424-447 (2017).

5. Corces-Zimmerman, M. R., Hong, W.-J., Weissman, I. L., Medeiros, B. C. \& Majeti, R. Preleukemic mutations in human acute myeloid leukemia affect epigenetic regulators and persist in remission. Proc. Natl Acad. Sci. USA 111, 2548-2553 (2014).

6. Papaemmanuil, E. et al. Genomic classification and prognosis in acute myeloid leukemia. N. Engl. J. Med. 374, 2209-2221 (2016).

7. Krönke, J. et al. Clonal evolution in relapsed NPM1-mutated acute myeloid leukemia. Blood 122, 100-108 (2013).

8. Suzuki, T. et al. Clinical characteristics and prognostic implications of NPM1 mutations in acute myeloid leukemia. Blood 106, 2854-2861 (2005).

9. Papadaki, C. et al. Monitoring minimal residual disease in acute myeloid leukaemia with NPM1 mutations by quantitative PCR: clonal evolution is a limiting factor. Br. J. Haematol. 144, 517-523 (2009).

10. Jan, M. et al. Clonal evolution of preleukemic hematopoietic stem cells precedes human acute myeloid leukemia. Sci. Transl. Med. 4, 149ra118 (2012).

11. Shlush, L. I. et al. Identification of pre-leukaemic haematopoietic stem cells in acute leukaemia. Nature 506, 328-333 (2014).

12. Jaiswal, S. et al. Age-related clonal hematopoiesis associated with adverse outcomes. N. Engl. J. Med. 371, 2488-2498 (2014).

13. Genovese, G. et al. Clonal hematopoiesis and blood-cancer risk inferred from blood DNA sequence. N. Engl. J. Med. 371, 2477-2487 (2014).

14. McKerrell, T. et al. Leukemia-associated somatic mutations drive distinct patterns of age-related clonal hemopoiesis. Cell Rep. 10, 1239-1245 (2015).

15. Bullinger, L., Döhner, K. \& Döhner, H. Genomics of acute myeloid leukemia diagnosis and pathways. J. Clin. Oncol. 35, 934-946 (2017).

16. Mullighan, C. G. et al. Genomic analysis of the clonal origins of relapsed acute lymphoblastic leukemia. Science 322, 1377-1380 (2008).

17. Ding, L. et al. Clonal evolution in relapsed acute myeloid leukaemia revealed by whole-genome sequencing. Nature. https://doi.org/10.1038/nature10738 (2012).

18. Wong, T. N. et al. Role of TP53 mutations in the origin and evolution of therapy-related acute myeloid leukaemia. Nature 518, 552-555 (2015).

19. Verhaak, R. G. W. et al. Mutations in nucleophosmin (NPM1) in acute myeloid leukemia (AML): association with other gene abnormalities and previously established gene expression signatures and their favorable prognostic significance. Blood 106, 3747-3754 (2005).

20. Wang, Y. et al. The Wnt $/ \beta$-catenin pathway is required for the development of leukemia stem cells in AML. Science 327, 1650-1653 (2010).

21. Feurstein, S. et al. Haploinsufficiency of ETV6 and CDKN1B in patients with acute myeloid leukemia and complex karyotype. BMC Genomics 15, 784 (2014).

22. Koenigsmann, J. et al. Nf1 haploinsufficiency and Icsbp deficiency synergize in the development of leukemias. Blood 113, 4690-4701 (2009).

23. Challen, G. A. et al. Dnmt3a is essential for hematopoietic stem cell differentiation. Nat. Genet. 44, 23-31 (2011).

24. Xie, M. et al. Age-related mutations associated with clonal hematopoietic expansion and malignancies. Nat. Med. 20, 1472-1478 (2014).

25. Gaidzik, V. I. et al. DNMT3A mutant transcript levels persist in remission and do not predict outcome in patients with acute myeloid leukemia. Leukemia 32, 30-37 (2018)

26. Yoshizato, T. et al. Somatic mutations and clonal hematopoiesis in aplastic anemia. N. Engl. J. Med. 373, 35-47 (2015). 
27. Lindsley, R. C. et al. Acute myeloid leukemia ontogeny is defined by distinct somatic mutations. Blood 125, 1367-1376 (2015).

28. Network, T. C. G. A. R. Comprehensive genomic characterization of squamous cell lung cancers. Nature 489, 519-525 (2012).

29. Hodis, E. et al. A landscape of driver mutations in melanoma. Cell $\mathbf{1 5 0}$ 251-263 (2012).

30. Saarinen, S. et al. Exome sequencing reveals germline NPAT mutation as a candidate risk factor for Hodgkin lymphoma. Blood 118, 493-498 (2011).

31. Falini, B. et al. Cytoplasmic nucleophosmin in acute myelogenous leukemia with a normal karyotype. N. Engl. J. Med. 352, 254-266 (2005).

32. Höllein, A. et al. NPM1 mutated AML can relapse with wild-type NPM1: persistent clonal hematopoiesis can drive relapse. Blood Adv. 2, 3118-3125 (2018).

33. Ivey, A. et al. Assessment of minimal residual disease in standard-risk AML. N. Engl. J. Med. 374, 422-433 (2016).

34. Krönke, J. et al. Monitoring of minimal residual disease in NPM1-mutated acute myeloid leukemia: a study from the German-Austrian acute myeloid leukemia study group. J. Clin. Oncol. 29, 2709-2716 (2011).

35. Lu, R. et al. Epigenetic perturbations by arg882-mutated dnmt3a potentiate aberrant stem cell gene-expression program and acute leukemia development. Cancer Cell 30, 92-107 (2016).

36. Thiede, C. et al. Rapid and sensitive typing of NPM1 mutations using LNAmediated PCR clamping. Leukemia 20, 1897-1899 (2006).

37. Gaidzik, V. I. et al. Clinical impact of DNMT3A mutations in younger adult patients with acute myeloid leukemia: results of the AML Study Group (AMLSG). Blood 121, 4769-4777 (2013).

38. Fröhling, S. et al. Prognostic significance of activating FLT3 mutations in younger adults (16 to 60 years) with acute myeloid leukemia and normal cytogenetics: a study of the AML Study Group Ulm. Blood 100, 4372-4380 (2002).

39. Paschka, P. et al. ASXL1 mutations in younger adult patients with acute myeloid leukemia: a study by the German-Austrian Acute Myeloid Leukemia Study Group. Haematologica 100, 324-330 (2015).

40. Paschka, P. et al. IDH1 and IDH2 mutations are frequent genetic alterations in acute myeloid leukemia and confer adverse prognosis in cytogenetically normal acute myeloid leukemia with npm1 mutation without FLT3 internal tandem duplication. J. Clin. Oncol. 28, 3636-3643 (2010).

41. Rücker, F. G. et al. TP53 alterations in acute myeloid leukemia with complex karyotype correlate with specific copy number alterations, monosomal karyotype, and dismal outcome. Blood. https://doi.org/10.1182/blood-201108-375758 (2011).

42. Paschka, P. et al. Secondary genetic lesions in acute myeloid leukemia with inv (16) or $\mathrm{t}(16 ; 16)$ : a study of the German-Austrian AML Study Group (AMLSG). Blood 121, 170-177 (2013).

43. Döhner, K. et al. Prognostic significance of partial tandem duplications of the MLL gene in adult patients 16 to 60 years old with acute myeloid leukemia and normal cytogenetics: a study of the acute myeloid leukemia study group Ulm. J. Clin. Oncol. 20, 3254-3261 (2002).

44. Li, H. Aligning sequence reads, clone sequences and assembly contigs with BWA-MEM. Preprint at: https://arxiv.org/abs/1303.3997 (2013)

45. McKenna, A. et al. The genome analysis toolkit: a mapreduce framework for analyzing next-generation DNA sequencing data. Genome Res. 20, 1297-1303 (2010).

46. Koboldt, D. C. et al. VarScan 2: somatic mutation and copy number alteration discovery in cancer by exome sequencing. Genome Res. 22, 568-576 (2012).

47. Wang, K., Li, M. \& Hakonarson, H. ANNOVAR: functional annotation of genetic variants from high-throughput sequencing data. Nucleic Acids Res. 38, e164-e164 (2010).

48. Sherry, S. T. et al. dbSNP: the NCBI database of genetic variation. Nucleic Acids Res. 29, 308-311 (2001).

49. The 1000 Genomes Project Consortium. A global reference for human genetic variation. Nature 526, 68-74 (2015).

50. Forbes, S. A. et al. COSMIC: exploring the world's knowledge of somatic mutations in human cancer. Nucleic Acids Res. 43, D805-D811 (2015).

51. Subramanian, A. et al. Gene set enrichment analysis: A knowledge-based approach for interpreting genome-wide expression profiles. Proc. Natl Acad. Sci. USA 102, 15545-15550 (2005).

52. Schroeder, C. M. et al. A comprehensive quality control workflow for paired tumor-normal NGS experiments. Bioinformatics 33, 1721-1722 (2017).

53. Quinlan, A. R. \& Hall, I. M. BEDTools: a flexible suite of utilities for comparing genomic features. Bioinformatics 26, 841-842 (2010).

54. Li, H. et al. The sequence alignment/map format and SAMtools. Bioinformatics 25, 2078-2079 (2009).

55. Zobeck, K. L. et al. 36-HaloPlexHS utilizes molecular barcodes to improve low frequency allele detection. Cancer Genet. 209, 296 (2016).
56. Dobin, A. et al. STAR: ultrafast universal RNA-seq aligner. Bioinformatics bts635. https://doi.org/10.1093/bioinformatics/bts635 (2012).

57. Love, M. I., Huber, W. \& Anders, S. Moderated estimation of fold change and dispersion for RNA-seq data with DESeq2. Genome Biol. 15, 550 (2014).

58. Simon, R. et al. Analysis of Gene Expression Data Using BRB-Array Tools. Cancer Inform. 3, 11-17 (2007).

59. Hoon, M. J. L., de Imoto, S., Nolan, J. \& Miyano, S. Open source clustering software. Bioinformatics 20, 1453-1454 (2004).

60. Eisen, M. B., Spellman, P. T., Brown, P. O. \& Botstein, D. Cluster analysis and display of genome-wide expression patterns. Proc. Natl Acad. Sci. USA 95, 14863-14868 (1998).

61. Heidel, F. H. et al. Genetic and pharmacologic inhibition of $\beta$-catenin targets imatinib-resistant leukemia stem cells in CML. Cell Stem Cell 10, 412-424 (2012).

62. R Development Core Team (2009) R: A Language and Environment for Statistical Computing. R Foundation for Statistical Computing, Vienna. http:// www.R-project.org. (2009).

63. Edgar, R., Domrachev, M. \& Lash, A. E. Gene expression omnibus: NCBI gene expression and hybridization array data repository. Nucleic Acids Res. 30, 207-210 (2002).

\section{Acknowledgements}

This study was supported in part by the Deutsche Forschungsgemeinschaft (SFB 1074 project B3 to KD and LB, project Z1 to HAK, and Heisenberg-Professur BU 1339/8-1 to LB) and a grant by the Else Kröner Fresenius Stiftung (2014_A298 to SK and KD). FHH was supported by the Thuringian state program ProExzellenz (RegenerAging - FSU-I-03/ 14) of the Thuringian Ministry for Research (TMWWDG).

\section{Author contributions}

S.C.: performed research, analyzed and interpreted data, wrote the manuscript. A.D.: performed research, analyzed, and interpreted data. S.K.-S.: performed research, analyzed data. F.G.R.: analyzed and interpreted data. S.L.: performed research, analyzed, and interpreted data. T.J. Blätte: analyzed and interpreted data. S.S.: performed experiments. J.K.: provided data, analyzed, and interpreted data. F.H.H.: performed experiments. T.M.S.: performed experiments. A.C.: performed experiments. V.I.G.: performed experiments. P.P.: performed experiments. V.T.: performed experiments. G.G.: provided reagents. F.T.: provided reagents. M.H.: provided reagents. A.G.: provided reagents. D.W.: analyzed data. E.S.: analyzed data. H.A.K.: analyzed data. H.D.: designed research, interpreted data. L.B.: designed research, analyzed, and interpreted data, wrote the manuscript. K.D.: designed research, analyzed, and interpreted data, wrote the manuscript.

\section{Additional information}

Supplementary Information accompanies this paper at https://doi.org/10.1038/s41467 019-09745-2.

Competing interests: The authors declare no competing interests.

Reprints and permission information is available online at http://npg.nature.com/ reprintsandpermissions/

Journal peer review information: Nature Communications thanks the anonymous reviewers for their contribution to the peer review of this work.

Publisher's note: Springer Nature remains neutral with regard to jurisdictional claims in published maps and institutional affiliations.

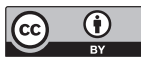

Open Access This article is licensed under a Creative Commons Attribution 4.0 International License, which permits use, sharing, adaptation, distribution and reproduction in any medium or format, as long as you give appropriate credit to the original author(s) and the source, provide a link to the Creative Commons license, and indicate if changes were made. The images or other third party material in this article are included in the article's Creative Commons license, unless indicated otherwise in a credit line to the material. If material is not included in the article's Creative Commons license and your intended use is not permitted by statutory regulation or exceeds the permitted use, you will need to obtain permission directly from the copyright holder. To view a copy of this license, visit http://creativecommons.org/ licenses/by/4.0/

(C) The Author(s) 2019 